\title{
On Some Approximation Theorems for Power $q$-Bounded Operators on Locally Convex Vector Spaces
}

\author{
Ludovic Dan Lemle \\ Department of Electrical Engineering and Industrial Informatics, Politehnica University of Timisoara, 331128 Hunedoara, Romania \\ Correspondence should be addressed to Ludovic Dan Lemle; dan.lemle@fih.upt.ro
}

Received 21 May 2014; Accepted 28 July 2014; Published 18 August 2014

Academic Editor: Antonio M. Peralta

Copyright (C) 2014 Ludovic Dan Lemle. This is an open access article distributed under the Creative Commons Attribution License, which permits unrestricted use, distribution, and reproduction in any medium, provided the original work is properly cited.

This paper deals with the study of some operator inequalities involving the power $q$-bounded operators along with the most known properties and results, in the more general framework of locally convex vector spaces.

\section{Introduction}

Let $X$ be a Hausdorff locally convex vector space over the complex field $\mathbb{C}$. By calibration for the locally convex space $X$ we understand a family $\mathscr{P}$ of seminorms generating the topology $\tau_{\mathscr{P}}$ of $X$, in the sense that this topology is the coarsest with respect to the fact that all the seminorms in $\mathscr{P}$ are continuous. Such a family of seminorms was used by the author and $\mathrm{Wu}[1]$ and many others in different contexts (see [2-5])

It is well known that calibration $\mathscr{P}$ is characterized by the property that the set

$$
E(p, \epsilon)=\{x \in X: p(x)<\epsilon\}, \quad \epsilon>0, p \in \mathscr{P}
$$

is a neighborhood subbase at 0 . Denote by $(X, \mathscr{P})$ the locally convex space $X$ endowed with calibration $\mathscr{P}$.

Recall that a locally convex algebra is an algebra with a locally convex topology in which the multiplication is separately continuous. Such an algebra is said to be locally $m$-convex (l.m.c.) if it has a neighborhood base $\mathcal{U}$ at 0 such that each $U \in \mathscr{U}$ is convex and balanced (i.e., $\lambda U \subseteq U$ for $|\lambda| \leq 1)$ and satisfies the property $U^{2} \subseteq U$.

Any algebra with identity will be called unital. It is well known that unital locally $m$-convex algebra $\mathscr{A}$ is characterized by the existence of calibration $\mathscr{P}$ such that each $p \in \mathscr{P}$ is submultiplicative (i.e., $p(x y) \leq p(x) p(y)$, for all $x, y \in \mathscr{A})$ and satisfies $p(e)=1$, where $e$ is the unit element.

An element $a$ of locally convex algebra $\mathscr{A}$ is said to be bounded in $\mathscr{A}$ if there exists $\alpha \in \mathbb{C}$ such that the set $\left\{(\alpha x)^{n}\right\}_{n \geq 1}$ is bounded in $\mathscr{A}$ (see [6]). The set of all bounded elements in $\mathscr{A}$ will be denoted by $\mathscr{A}_{0}$.

Let $\mathbb{C}_{\infty}:=\mathbb{C} \cup\{\infty\}$ be the Alexandroff one-point compactification of $\mathbb{C}$. Following Waelbroeck $[7,8]$, we introduce the following.

Definition 1. We call resolvent set in the Waelbroeck sense of an element $x$ from a locally convex unital algebra $(X, \mathscr{P})$ the set of all elements $\lambda_{0} \in \mathbb{C}_{\infty}$ for which there exists $V \in \mathscr{V}_{\lambda_{0}}$ such that the following conditions hold:

(a) the element $\lambda e-x$ is invertible in $X$, for any $\lambda \in V$ $\{\infty\}$;

(b) the set $\left\{(\lambda e-x)^{-1}: \lambda \in V \backslash\{\infty\}\right\}$ is bounded in $(X, \mathscr{P})$.

The resolvent set in Waelbroeck sense of an element $x$ will be denoted by $\rho_{W}(x)$. The Waelbroeck spectrum of $x$ will be defined as

$$
\sigma_{W}(x):=\mathbb{C}_{\infty} \backslash \rho_{W}(x)
$$




\section{2. $q$-Bounded Operators}

Following Michael [9] (see also [2, 10]), we introduce the following.

Definition 2. We say that a linear operator $T: X \rightarrow X$ is $q$-bounded (quotient-bounded) with respect to $\mathscr{P}$ if for any $p \in \mathscr{P}$ there exists $c_{p}>0$ such that

$$
p(T x) \leq c_{p} p(x), \quad \forall x \in X
$$

Denote by $Q_{\mathscr{P}}(X)$ the set which consists of all $q$-bounded operators with respect to calibration $\mathscr{P}$.

For a seminorm $p \in \mathscr{P}$, the application $\widehat{p}: Q_{\mathscr{P}}(X) \rightarrow \mathbb{R}$ defined as

$$
\widehat{p}(T)=\inf \{r>0: p(T x) \leq r p(x), \forall x \in X\}
$$

is also a seminorm. Note that

$$
\widehat{p}\left(T_{1} T_{2}\right) \leq \widehat{p}\left(T_{1}\right) \widehat{p}\left(T_{2}\right), \quad T_{1}, T_{2} \in Q_{\mathscr{P}}(X), p \in \mathscr{P} .
$$

We denote by $\widehat{\mathscr{P}}$ the family of seminorms $\{\widehat{p}: p \in \mathscr{P}\}$. The space $Q_{\mathscr{P}}(X)$ will be endowed with a topology $\tau_{\widehat{\mathscr{P}}}$ generated by $\widehat{\mathscr{P}}$. Remark that $[9$, Proposition $2.4(\mathrm{j})]$ implies that under this topology $Q_{\mathscr{P}}(X)$ becomes a Hausdorff locally $m$-convex topological algebra (in the sense of [9, Definition 2.1]).

If $T \in Q_{\mathscr{P}}(X)$, the $\mathscr{P}$-spectral radius, denoted by $r_{\mathscr{P}}(T)$, is considered as the boundedness radius in the sense of Allan [6] (see also [11-13]),

$$
\begin{aligned}
& r_{\mathscr{P}}(T)=\inf \left\{\lambda>0: \text { the sequence }\left(\left(\lambda^{-1} T\right)^{n}\right)_{n \in \mathbb{N}}\right. \\
&\text { is bounded in } \left.Q_{\mathscr{P}}(X)\right\},
\end{aligned}
$$

where, by common consent, inf $\emptyset:=+\infty$.

The set of all bounded elements in $Q_{\mathscr{P}}(X)$ will be denoted by $\left(Q_{\mathscr{P}}(X)\right)_{0}$ (see [12]). It easily follows from [6, Proposition 2.14(ii)] that

$$
\left(Q_{\mathscr{P}}(X)\right)_{0}=\left\{T \in Q_{\mathscr{P}}(X): r_{\mathscr{P}}(T)<\infty\right\}
$$

For $T \in\left(Q_{\mathscr{P}}(X)\right)_{0}$ we denote by $\rho_{W}(T)$ the Waelbroeck resolvent set of $T$ and by $\sigma_{W}(T)$ the Waelbroeck spectrum of $T$. The function

$$
\rho_{W}(T) \ni \lambda \longmapsto R(\lambda, T):=(\lambda I-T)^{-1} \in\left(Q_{\mathscr{P}}(X)\right)_{0}
$$

is called the resolvent function of $T$. It is well known that

$$
R(\lambda, T)=\sum_{n=0}^{\infty} \frac{T^{n}}{\lambda^{n+1}}
$$

In this paper we evaluate the behaviour of the power of a $q$-bounded operator from the algebra $\left(Q_{\mathscr{P}}(X)\right)_{0}$ by some type of approximations. The main results have been announced in [14].

\section{The Main Results}

We continue to employ the notations from the previous sections and we will introduce two types of operatorial approximations for operators from the algebra $\left(Q_{\mathscr{P}}(X)\right)_{0}$ which approximate a given operator $T$ on a convergent power bounded series. The power boundedness problem for operators acting on Banach spaces was largely developed in various frameworks by many authors (see [15-17]).

In the following, using the functional calculus from the $\left(Q_{\mathscr{P}}(X)\right)_{0}$ algebra (see $\left.[7,8]\right)$, some important boundedness properties are obtained. Denote $\mathbb{N}^{*}=\mathbb{N} \backslash\{0\}$. First we have the following.

Theorem 3. If $T \in\left(Q_{\mathscr{P}}(X)\right)_{0}$ satisfies

$$
\sup _{\widehat{p} \in \widehat{P}} \widehat{p}\left(T^{k}\right) \leq C,
$$

for $k \in \mathbb{N}^{*}$, then

$$
\sup _{\widehat{p} \in \widehat{P}} \widehat{P}\left[R(\lambda, T)^{k}\right] \leq \frac{C}{(|\lambda|-1)^{k}},
$$

for $k \in \mathbb{N}^{*}$ and for all $\lambda \in \mathbb{C}$ with $|\lambda|>1$.

Proof. Assume that $\sup _{\widehat{p} \in \widehat{\mathscr{P}}} \widehat{p}\left(T^{k}\right) \leq C$ for $k \in \mathbb{N}^{*}$. Since

$$
R(\lambda, T)=\sum_{j=0}^{\infty} \frac{T^{j}}{\lambda^{j+1}}
$$

for $|\lambda|>1$, then, by using the generalized binomial formula, we get

$$
R(\lambda, T)^{k}=\lambda^{-k}\left(I-\frac{T}{\lambda}\right)^{-k}=\frac{1}{\lambda^{k}} \sum_{j=0}^{\infty}\left(\begin{array}{c}
j+k-1 \\
j
\end{array}\right) \frac{T^{j}}{\lambda^{j}},
$$

from where we deduce

$$
\begin{aligned}
\widehat{p}\left[R(\lambda, T)^{k}\right] & \leq \frac{C}{|\lambda|^{k}} \cdot \sum_{j=0}^{\infty}\left(\begin{array}{c}
j+k-1 \\
j
\end{array}\right)\left(\frac{1}{|\lambda|}\right)^{j} \\
& =\frac{C}{|\lambda|^{k}} \cdot \frac{1}{(1-1 /|\lambda|)^{k}}=\frac{C}{(|\lambda|-1)^{k}},
\end{aligned}
$$

for any $k \in \mathbb{N}^{*}$ and any $\widehat{p} \in \widehat{\mathscr{P}}$. Therefore, the conclusion is verified.

Conversely, we have the following.

Theorem 4. If $T \in\left(Q_{\mathscr{P}}(X)\right)_{0}$ and

$$
\sup _{\widehat{p} \in \widehat{P}} \widehat{P}[R(\lambda, T)] \leq \frac{C}{|\lambda|-1},
$$

for all $\lambda \in \mathbb{C}$ with $|\lambda|>1$, then

$$
\sup _{\widehat{p} \in \widehat{P}} \widehat{P}\left(T^{k}\right) \leq C e(k+1)
$$

for $k \in \mathbb{N}^{*}$. 
Proof. Let us suppose condition $\widehat{p}[R(\lambda, T)] \leq C /(|\lambda|-1)$ is true for all $\widehat{p} \in \widehat{\mathscr{P}}$, for any $k \in \mathbb{N}^{*}$ and $|\lambda|>1$. For $k \in \mathbb{N}^{*}$ fixed, by choosing the integration path $\Gamma:|\lambda|=1+1 / k$, with the aid of the functional calculus from the algebra $\left(Q_{\mathscr{P}}(X)\right)_{0}$, we obtain

$$
T^{k}=\frac{1}{2 \pi i} \int_{\Gamma} \lambda^{k} R(\lambda, T) d \lambda .
$$

Thus, for all $\widehat{p} \in \widehat{\mathscr{P}}$, we have

$$
\begin{aligned}
\widehat{p}\left(T^{k}\right) & \leq \frac{1}{2 \pi} \int_{\Gamma}|\lambda|^{k} \widehat{p}(R(\lambda, T)) d \lambda \\
& \leq \frac{1}{2 \pi} \cdot \max _{\lambda \in \Gamma}|\lambda|^{k} \cdot \max _{\lambda \in \Gamma} \frac{C}{|\lambda|-1} \cdot \int_{\Gamma} d \lambda \\
& \leq \frac{1}{2 \pi} \cdot\left(1+\frac{1}{k}\right)^{k} \cdot C k \cdot 2 \pi\left(1+\frac{1}{k}\right) \leq C e(k+1)
\end{aligned}
$$

which implies the desired result.

Moreover, we can formulate the following.

Theorem 5. If $T \in\left(Q_{\mathscr{P}}(X)\right)_{0}$ and

$$
\sup _{\widehat{p} \in \mathscr{P}}\left[R(\lambda, T)^{k}\right] \leq \frac{C}{(|\lambda|-1)^{k}},
$$

for $k \in \mathbb{N}^{*}$ and for all $\lambda \in \mathbb{C}$ with $|\lambda|>1$, then

$$
\sup _{\widehat{p} \in \widehat{P}}\left(T^{k}\right) \leq C \frac{k ! e^{k}}{k^{k}} \leq C \sqrt{2 \pi(k+1)}, \quad k \in \mathbb{N}^{*} .
$$

Proof. Integrating $(*)$ by parts $j-1$ times, for $j>2$, we obtain

$$
T^{k}=\frac{(-1)^{j-1}}{2 \pi i} \int_{\Gamma} \frac{(j+1) ! \lambda^{k+j-1}}{(k+1) \cdots(k+j-1)} R(\lambda, T)^{j} d \lambda .
$$

Now choosing $\Gamma$ the circle of radius $1+j / k$ and by using the hypothesis, for $j \rightarrow \infty$, we get

$$
\sup _{\widehat{p} \in \widehat{P}} \widehat{P}\left(T^{k}\right) \leq C \frac{k ! e^{k}}{k^{k}} \leq C \sqrt{2 \pi(k+1)} .
$$

The last inequality was obtained by using Stirling's approximation.

Now, for $T \in\left(Q_{\mathscr{P}}(X)\right)_{0}$ we introduce (see [18]) the following.

Definition 6. The Yosida approximation $Y(\lambda, T)$ of $T$, for $\lambda \epsilon$ $\rho_{W}(T) \cap \mathbb{C}$, is defined as

$$
Y(\lambda, T)=\lambda T R(\lambda, T) .
$$

Next theorem shows how an operator from the $\left(Q_{\mathscr{P}}(X)\right)_{0}$ algebra is related to its Yosida approximation.

Theorem 7. The Yosida approximation $Y(\lambda, T)$ is analytic for $\lambda \in \rho_{W}(T) \cap \mathbb{C}$ and the series representation

$$
Y(\lambda, T)=\sum_{j=0}^{\infty} \frac{T^{j+1}}{\lambda^{j}}
$$

converges for $|\lambda|>r_{\mathscr{P}}(T)$. Moreover,
(1) $Y(\lambda, T)=\lambda^{2} R(\lambda, T)-\lambda I$;

(2) if there exists $\widehat{p} \in \widehat{\mathscr{P}}$ such that $r_{\mathscr{P}}(T)<\widehat{p}(T)$, then

$$
\widehat{p}(Y(\lambda, T)-T) \leq \frac{\widehat{p}\left(T^{2}\right)}{|\lambda|-\widehat{p}(T)},
$$

for $|\lambda|>\widehat{p}(T)$;

(3) $\sigma_{W}(Y(\lambda, T))=\left\{z /(1-z / \lambda), z \in \sigma_{W}(T)\right\}$.

Proof. By evaluating $Y(\lambda, T)$ in terms of the resolvent $R(\lambda, T)$, for $|\lambda|>r_{\mathscr{P}}(T)$ we obtain

$$
\begin{aligned}
Y(\lambda, T) & =\lambda T R(\lambda, T)=\lambda T(\lambda I-T)^{-1} \\
& =\lambda T \cdot \sum_{j=0}^{\infty} \frac{T^{j}}{\lambda^{j+1}}=\sum_{j=0}^{\infty} \frac{T^{j+1}}{\lambda^{j}}
\end{aligned}
$$

from where it follows that the assertion of the theorem is true. Moreover,

$$
\begin{aligned}
Y(\lambda, T) & =\sum_{j=0}^{\infty} \frac{T^{j+1}}{\lambda^{j}}=\lambda I+T+\frac{T^{2}}{\lambda}+\cdots+\frac{T^{n+1}}{\lambda^{n}}+\cdots-\lambda I \\
& =\lambda^{2} \sum_{j=0}^{\infty} \frac{T^{j}}{\lambda^{j+1}}-\lambda I=\lambda^{2} R(\lambda, T)-\lambda I,
\end{aligned}
$$

so (1) is true.

To prove (2) one can observe that, from

$$
Y(\lambda, T)=\sum_{j=0}^{\infty} \frac{T^{j+1}}{\lambda^{j}},
$$

it follows that

$$
Y(\lambda, T)-T=\sum_{j=0}^{\infty} \frac{T^{2}}{\lambda}\left(\frac{T^{j}}{\lambda^{j}}\right)
$$

on a set for which $|\lambda|>r_{\mathscr{P}}(T)$. Moreover,

$$
\begin{aligned}
\widehat{p}(Y(\lambda, T)-T) & \leq \sum_{j=0}^{\infty} \widehat{p}\left(\frac{T^{2}}{\lambda}\right) \widehat{p}\left(\frac{T^{j}}{\lambda^{j}}\right) \\
& \leq \widehat{p}\left(\frac{T^{2}}{\lambda}\right) \cdot \sum_{j=0}^{\infty} \hat{p}\left(\frac{T}{\lambda}\right)^{j} \\
& =\widehat{p}\left(\frac{T^{2}}{\lambda}\right) \frac{1}{1-\widehat{p}(T / \lambda)}=\frac{\widehat{p}\left(T^{2}\right)}{|\lambda|-\widehat{p}(T)},
\end{aligned}
$$

for $|\lambda|>\widehat{p}(T)>r_{\mathscr{P}}(T)$.

A simple reasoning shows that $R(\lambda, T) \in\left(Q_{\mathscr{P}}(X)\right)_{0}$; then it follows $Y(\lambda, T) \in\left(Q_{\mathscr{P}}(X)\right)_{0}$.

From [19, Theorem 3.1.14], for $|\lambda|>|z|$, we have

$$
\sigma_{W}(Y(\lambda, T))=Y\left(\lambda, \sigma_{W}(T)\right),
$$

for all $z \in \sigma_{W}(T)$, and

$$
Y(\lambda, z)=\sum_{j=0}^{\infty} \frac{z^{j+1}}{\lambda^{j}}
$$

on $|\lambda|>|z|$, which could be written as $Y(\lambda, z)=z /(1-z / \lambda)$, for any $z \in \sigma_{W}(T)$, so (3) is proved. 
Below we state an equivalence between a power bounded operator from the $\left(Q_{\mathscr{P}}(X)\right)_{0}$ algebra and the power of its Yosida approximation.

Theorem 8. Let $T \in\left(Q_{\mathscr{P}}(X)\right)_{0}$ and $Y(\lambda, T)$ its Yosida approximation. Then the following assertions are equivalent:

(i) $\sup _{\widehat{p} \in \widehat{\mathscr{P}}} \widehat{p}\left(T^{k}\right) \leq c$, for any $k \in \mathbb{N}^{*}$;

(ii) $\sup _{\widehat{p} \in \widehat{P}} \widehat{p}\left(Y(\lambda, T)^{k}\right) \leq c /(1-1 /|\lambda|)^{k}$, for any $k \in \mathbb{N}^{*}$ and for all $\lambda \in \mathbb{C}$ with $|\lambda|>1$.

Proof. Property (i) implies $r_{\mathscr{P}}(T) \leq 1$ so that the argumentation given in the proof of Theorem 7 implies that any $\lambda \in \mathbb{C}$ with $|\lambda|>1$ belongs to the resolvent set of $T$. Hence, using the generalized binomial formula, we get

$$
Y(\lambda, T)^{k}=\sum_{j=0}^{\infty}\left(\begin{array}{c}
k+j-1 \\
j
\end{array}\right) \frac{T^{j+k}}{\lambda^{j}} .
$$

Now, by applying (i) again we obtain

$$
\widehat{p}\left(Y(\lambda, T)^{k}\right) \leq c \sum_{j=0}^{\infty}\left(\begin{array}{c}
k+j-1 \\
j
\end{array}\right)\left(\frac{1}{|\lambda|}\right)^{j}=\frac{c}{(1-1 /|\lambda|)^{k}}
$$

for any $\widehat{p} \in \widehat{\mathscr{P}}$, whence by passing to supremum, the inequality (ii) holds.

Conversely, (i) is a direct consequence of (ii).

For $\mu \in \rho_{W}(T)$, consider now the following Möbius transformation (see [20]):

$$
\psi_{\lambda}(\mu)= \begin{cases}\frac{(\lambda-1) \mu}{\lambda-\mu}, & \text { if } \lambda \neq \infty \\ \mu, & \text { if } \lambda=\infty\end{cases}
$$

Definition 9. The Möbius approximation of $T$ is defined as

$$
A(\lambda, T):=\psi_{\lambda}(T) .
$$

Proposition 10. $A(\lambda, T)$ is holomorphic in $\lambda \in \rho_{W}(T) \cap \mathbb{C}$ and satisfies

$$
A(\lambda, T)=\left(1-\frac{1}{\lambda}\right) Y(\lambda, T), \quad \lambda \neq 0 .
$$

Proof. Let $\lambda \in \rho_{W}(T) \cap \mathbb{C} \backslash\{0\}$. By evaluating the right member of the above equality, we get successively

$$
\begin{aligned}
(1 & \left.-\frac{1}{\lambda}\right) Y(\lambda, T) \\
& =\left(1-\frac{1}{\lambda}\right) \lambda T R(\lambda, T)=(\lambda-1) T R(\lambda, T) \\
& =\frac{(\lambda-1) T}{\lambda I-T}=A(\lambda, T)
\end{aligned}
$$

for $\lambda \neq \infty$. If $\lambda=\infty$, then from Definition 9 we have $A(\lambda, T)=T$. On the other side $(1-1 / \lambda) Y(\lambda, T)$ converges to $T$, when $\lambda \rightarrow \infty$.
A similar result as in Theorem 8 is given below.

Theorem 11. Let $T \in\left(Q_{\mathscr{P}}(X)\right)_{0}$ and $A(\lambda, T)$ its approximation as above. Then the following assertions are equivalent:

(i) $\sup _{\widehat{p} \in \widehat{\mathscr{P}}} \widehat{p}\left(T^{k}\right) \leq C$, for any $k \in \mathbb{N}^{*}$;

(ii) $\sup _{\widehat{p} \in \widehat{P}} \widehat{p}\left(A(\lambda, T)^{k}\right) \leq C$, for any $k \in \mathbb{N}^{*}$ and for every $\lambda \in \mathbb{C}$ with $|\lambda|>1$.

Proof. From Theorem 8, for $T \in\left(Q_{\mathscr{P}}(X)\right)_{0}$,

$$
\sup _{\widehat{p} \in \widehat{P}} \widehat{p}\left(T^{k}\right) \leq C
$$

is equivalent to

$$
\sup _{\widehat{p} \in \widehat{P}} \widehat{P}\left(Y(\lambda, T)^{k}\right) \leq \frac{C}{(1-1 /|\lambda|)^{k}} .
$$

The conclusion follows taking into account that

$$
A(\lambda, T)^{k}=\left(1-\frac{1}{\lambda}\right)^{k} \cdot Y(\lambda, T)^{k}
$$

for $k \in \mathbb{N}^{*}$.

\section{Application}

For $L>0$ let $X:=\mathscr{C}[0, L]$ be the space of continuous functions on $[0, L]$ endowed with the norm $|u|_{L}:=\max _{[0, L]}|u(t)|$.

Consider $T: X \rightarrow X$, given by

$$
T u(t)=\int_{0}^{t} u(s) d s .
$$

Following [19], we see that the resolvent of $T$ is given by

$$
R(\lambda, T) u(t)=\frac{1}{\lambda} u(t)+\frac{1}{\lambda^{2}} \int_{0}^{t} e^{(t-s) / \lambda} u(s) d s,
$$

the Yosida approximation of $T$ is

$$
Y(\lambda, T) u(t)=\int_{0}^{t} e^{(t-s) / \lambda} u(s) d s,
$$

and the Möbius approximation of $T$ is

$$
A(\lambda, T) u(t)=\left(1-\frac{1}{\lambda}\right) \int_{0}^{t} e^{(t-s) / \lambda} u(s) d s .
$$

Remark that, for all $u \in \mathscr{C}[0, L]$, we have

$$
\begin{aligned}
|T u|_{L} & =\max _{t \in[0, L]}|T u(t)| \\
& \leq \max _{t \in[0, L]} \int_{0}^{t}|u(s)| d s \\
& \leq \max _{t \in[0, L]}|u(t)| \int_{0}^{L} d s=|u|_{L} \cdot L .
\end{aligned}
$$

The above implies that $T$ is a contraction for $L \leq 1$. 
If $L>1$, then we can introduce for each $\varepsilon>0$ the following norm on $\mathscr{C}[0, L]$ :

$$
\|u\|_{\mathcal{E}}:=\max _{t \in[0, L]} e^{t / \varepsilon}|u(t)|, \quad u \in \mathscr{C}[0, L] .
$$

Then a simple computation gives that

$$
\|T u\|_{\varepsilon}<\varepsilon\|u\|_{\varepsilon}, \quad u \in \mathscr{C}[0, L] .
$$

On the other hand,

$$
\|u\|_{\varepsilon} \leq|u|_{L} \leq e^{L / \varepsilon}\|u\|_{\varepsilon} .
$$

Remark that, by Theorem 11, for all $\lambda>1$, we get

$$
|A(\lambda, T)|_{L}=(\lambda-1)\left(e^{T / \lambda}-1\right) \leq 1
$$

if and only if $|T|_{L} \leq 1$.

It is clear that for estimating the powers of $T$ it seems to be better to use the Yosida approximation or Möbius approximation than the resolvent approximation.

\section{Conflict of Interests}

The author declares that there is no conflict of interests regarding the publication of this paper.

\section{Acknowledgment}

The author is grateful to the anonymous referees for their very careful reading and for useful suggestions that helped in better exposing this material.

\section{References}

[1] L. D. Lemle and L. M. Wu, "Uniqueness of $\mathrm{C}_{0}$-semigroups on a general locally convex vector space and an application," Semigroup Forum, vol. 82, no. 3, pp. 485-496, 2011.

[2] R. T. Moore, "Banach algebras of operators on locally convex spaces," Bulletin of the American Mathematical Society, vol. 75, pp. 68-73, 1969.

[3] F. Pater, "Properties of multipliers on special algebras with application to signal processing," Acta Technica Napocensis: Applied Mathematics and Mechanics, vol. 54, pp. 313-318, 2011.

[4] F. Pater, "A multiplier algebra representation with application to harmonic signal models," in Proceedings of the A.I.P. International Conference on Numerical Analysis and Applied Mathematics, vol. 1479, pp. 1075-1078, Kos, Greece, September 2012.

[5] M. Kostić, "Abstract Volterra equations in locally convex spaces," Science China: Mathematics, vol. 55, no. 9, pp. 17971825, 2012.

[6] G. R. Allan, "A spectral theory for locally convex alebras," Proceedings of the London Mathematical Society, vol. 15, pp. 399421, 1965.

[7] L. Waelbroeck, Étude Spectrale des Algèbres Complètes, vol. 31, Académie Royale de Belgique. Classe des sciences. Mémoires. Col., 1960.

[8] L. Waelbroeck, "Algébrés commutatives: éléments réguliers," Bulletin of the Belgian Mathematical Society, vol. 9, pp. 42-49, 1957.
[9] E. A. Michael, "Locally multiplicatively-convex topological algebras," Memoirs of the American Mathematical Society, vol. 1952, no. 11, 79 pages, 1952.

[10] G. A. Joseph, "Boundedness and completeness in locally convex spaces and algebras," Journal of the Australian Mathematical Society, vol. 24, no. 1, pp. 50-63, 1977.

[11] F. G. Bonales and R. V. Mendoza, "Extending the formula to calculate the spectral radius of an operator," Proceedings of the American Mathematical Society, vol. 126, no. 1, pp. 97-103, 1998.

[12] F. Pater and T. Binzar, "On some ergodic theorems for a universally bounded operator," Carpathian Journal of Mathematics, vol. 26, no. 1, pp. 97-102, 2010.

[13] F. Pater and L. D. Lemle, "On some multiplication operator algebra problem with application to stochastic signal models," in Proceedings of the International Conference on Numerical Analysis and Applied Mathematics, vol. 1558 of AIP Conference Proceedings, pp. 1661-1664, Rhodes, Greece, September 2013.

[14] F. Pater, L. D. Lemle, and T. Binzar, "On some Yosida type approximation theorems," in Proceedings of the International Conference on Numerical Analysis and Applied Mathematics, vol. 1168 of AIP Conference Proceedings, pp. 521-524, Rethymno, Crete, September 2009.

[15] Y. Katznelson and L. Tzafriri, "On power bounded operators," Journal of Functional Analysis, vol. 68, no. 3, pp. 313-328, 1986.

[16] B. Nagy and J. Zemanek, "A resolvent condition implying power boundedness," Studia Mathematica, vol. 134, no. 2, pp. 143-151, 1999.

[17] O. Nevanlinna, "On the growth of the resolvent operator for power bounded operators, Linear Operators," Banach Center Publications, vol. 38, pp. 247-264, 1997.

[18] K. Yosida, "On the differentiability and the representation of one-parameter semi-group of linear operators," Journal of the Mathematical Society of Japan, vol. 1, pp. 15-21, 1948.

[19] O. Nevanlinna, Convergence of Iterations for Linear Equations, Birkhäuser, Basel, Switzerland, 1993.

[20] A. L. Shields, "On Möbius bounded operators," Acta Universitatis Szegediensis: Acta Scientiarum Mathematicarum, vol. 40, no. 3-4, pp. 371-374, 1978. 


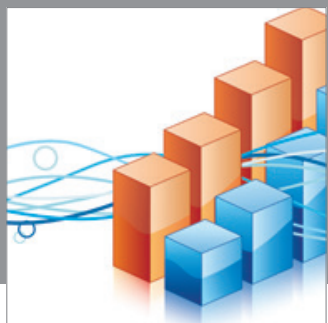

Advances in

Operations Research

mansans

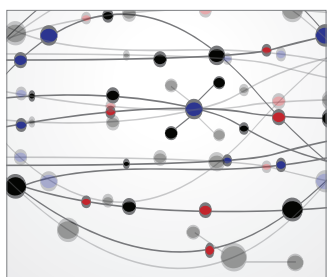

The Scientific World Journal
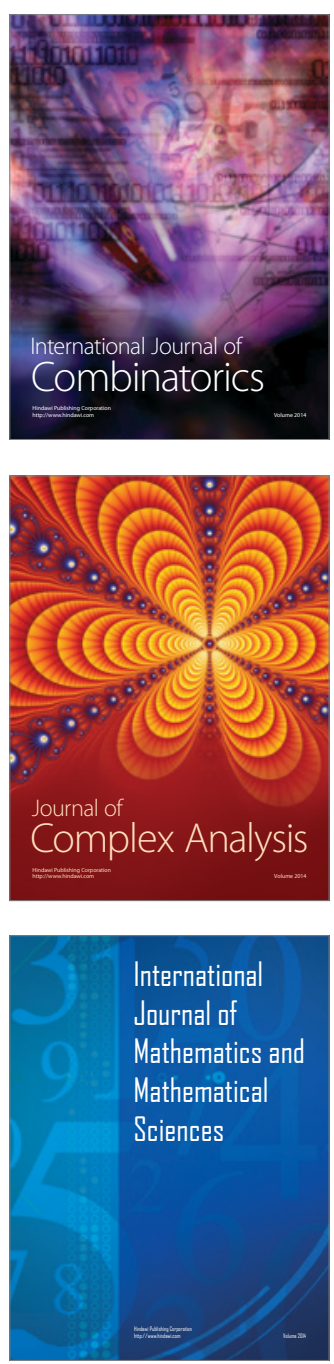
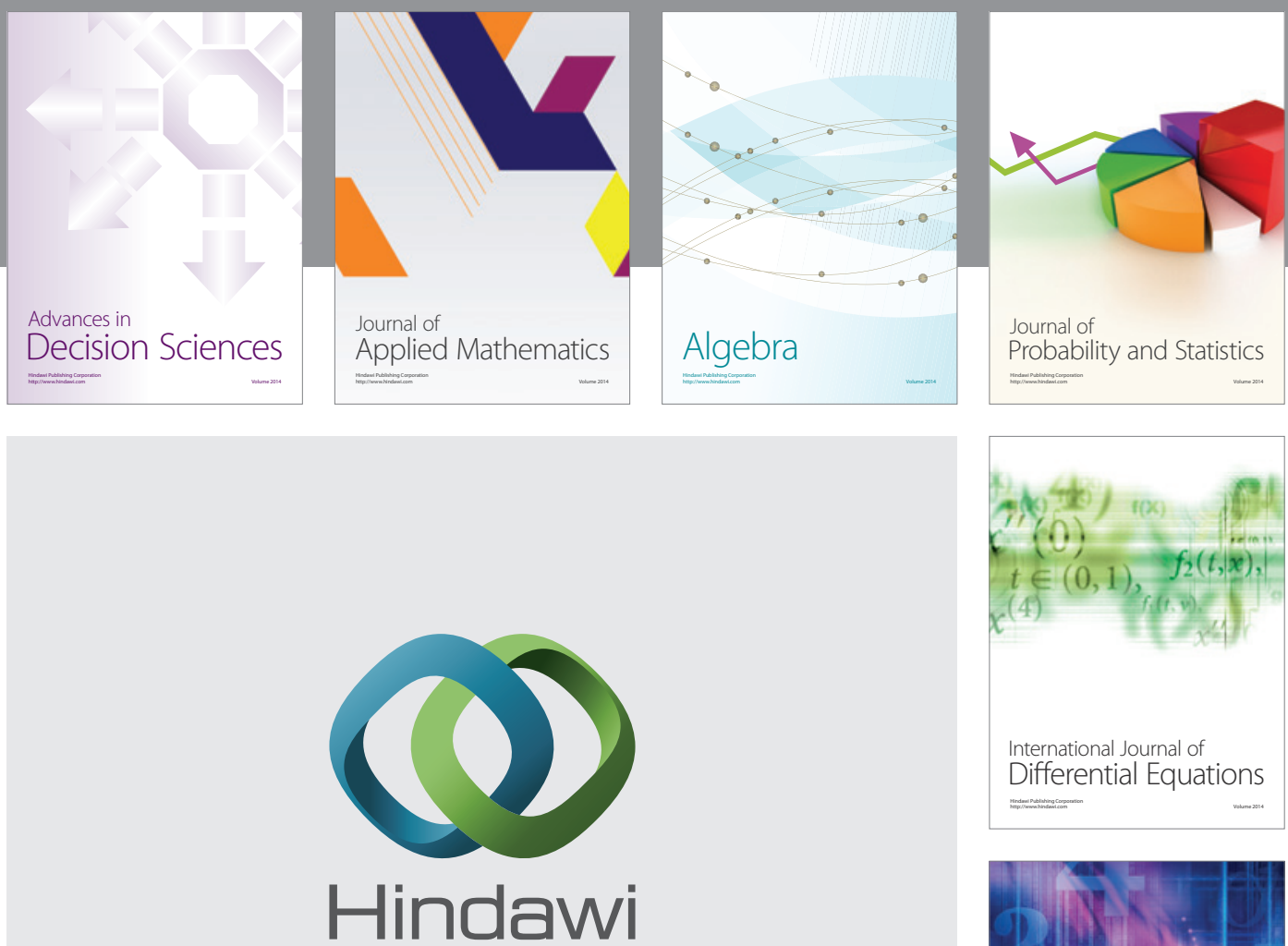

Submit your manuscripts at http://www.hindawi.com
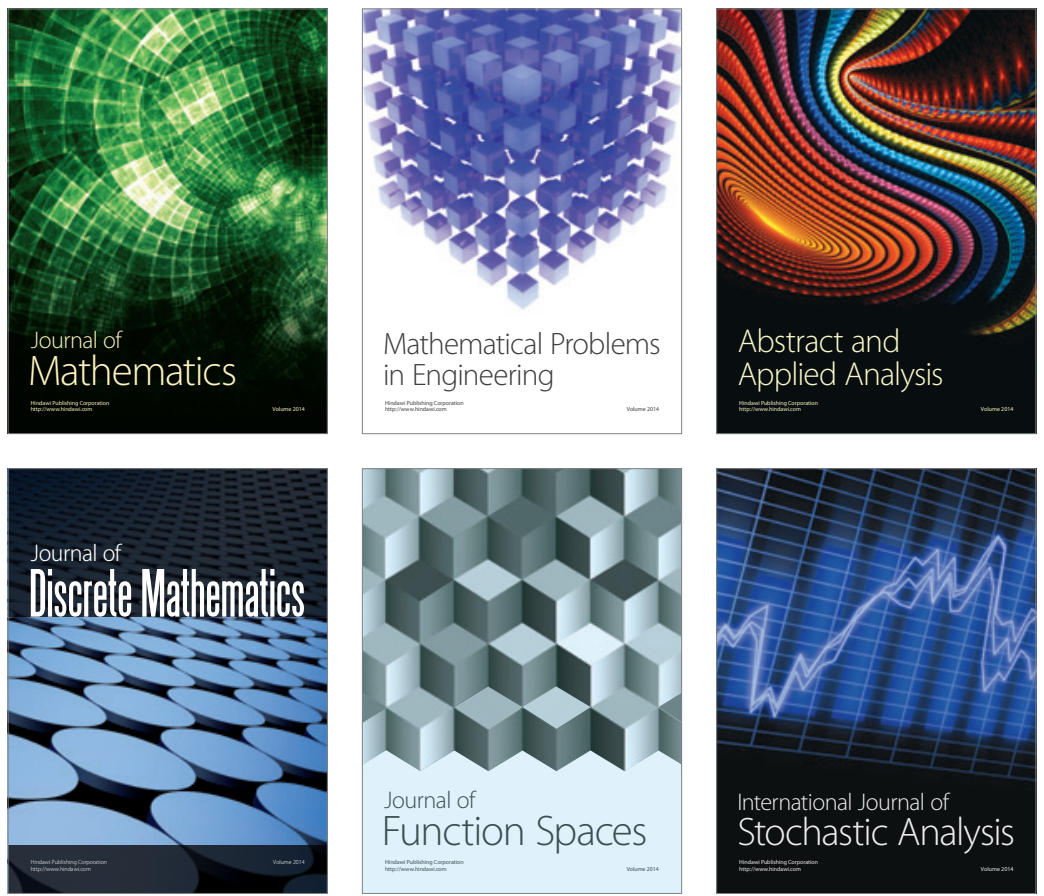

Journal of

Function Spaces

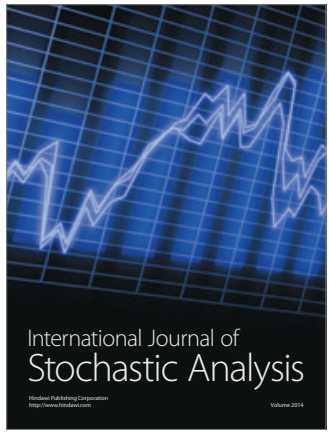

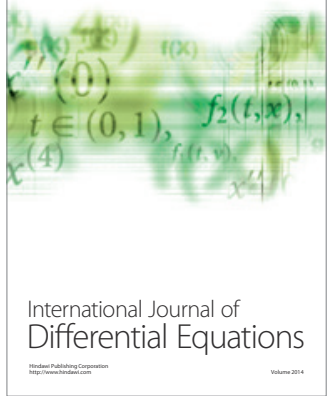
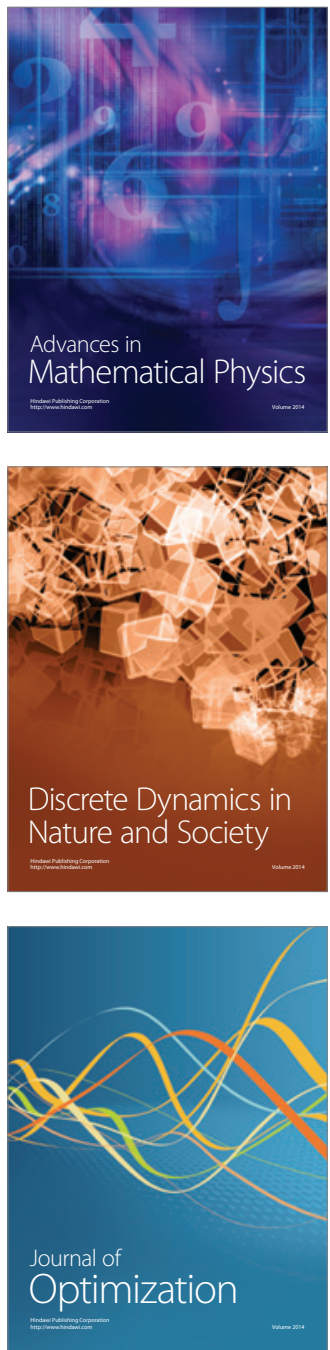